\section{- OPEN ACCESS}

\title{
A case of De Garengeot hernia and literature review
}

\author{
Bardia Bidarmaghz, ${ }^{1}$ Chin Li Tee²
}

${ }^{1}$ General Surgery, Caboolture Hospital, Caboolture, Queensland, Australia ${ }^{2}$ General Surgery, Caboolture Hospital, Caboolture, Queensland, Australia

\section{Correspondence to} Dr Bardia Bidarmaghz, bardiab1985@yahoo.com

Accepted 23 August 2017

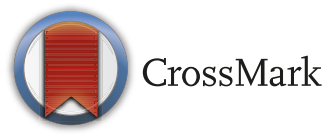

To cite: Bidarmaghz B, Tee CL. BMJ Case Rep Published Online First: [please include Day Month Year]. doi:10.1136/bcr-2017 220926

\section{SUMMARY}

Femoral hernia accounts for only $3 \%$ of all the hernias and in only $0.5 \%-5 \%$ of the events, the appendix can travel through the femoral hernia which is called De Garengeot hernia, and the incidence of appendicitis in this type of hernia is as low as $0.08 \%-0.13 \%$. We present a case of a 69-year-old healthy woman who was referred to the emergency department by her general practitioner for CT-proven appendicitis in the femoral canal. On initial assessment, she was found to have a hard, tender lump in her right groin below the inguinal ligament, and open appendectomy and herniorrhaphy were performed. Surgery is the mainstay of treatment of this type of hernia but due to the rarity of this condition, there is no specific guideline as for the surgical procedure. This article demonstrated a case of De Garengeot hernia which was diagnosed preoperatively and managed surgically.

\section{BACKGROUND}

Femoral hernia occurs as the result of protrusion of abdominal content through the femoral canal medial to the femoral artery and below the inguinal ring. This type of hernia is more common in women and accounts for only $3 \%$ of all the hernias and has $15 \%-20 \%$ chance of strangulation because of the narrow femoral neck. ${ }^{1}$ In only $0.5 \%-5 \%$ of the events, the appendix can travel through the femoral hernia which is called De Garengeot hernia, ${ }^{2}$ and the incidence of appendicitis in this type of hernia is as low as $0.08 \%-0.13 \%{ }^{3}$ De Garengeot hernia is a rare clinical presentation and there is no published guideline regarding the procedure of choice, ${ }^{4}$ and it must be differentiated from Amyand hernia, which is the presence of appendix inside an inguinal hernia. ${ }^{5}$ We present a case of preoperatively diagnosed De Garengeot hernia and review the literature to help the readers to have a better understanding of this case scenario.

\section{CASE PRESENTATION}

A 69-year-old healthy woman was referred by her general practitioner (GP) for CT-proven appendicitis present in right-sided femoral hernia (figure 1). For the past 7 days, she had noticed a lump in her right groin which was initially reducible and associated with mild discomfort on exertion, but it was getting more painful during the past 24 hours accompanied by low-grade fever. She denied nausea, vomiting and her bowel motion was normal. The patient saw her GP due to worsening of the pain, and an ultrasound was organised which demonstrated a right-sided femoral hernia below the inguinal ligament that contained the appendix. There was a small amount of fluid around the appendix and increased vascularity which was suggestive of appendicitis. CT was then ordered by patient's primary physician and the scan confirmed the presence of an inflamed appendix inside femoral hernia sac; therefore she was transferred to emergency department. She was an independent, non-smoker woman with no significant medical history apart from mild gastro-oesophageal reflux and vaginal hysterectomy 20 years ago.

\section{INVESTIGATIONS}

On arrival to the emergency department, she was complaining of discomfort in her right groin and had blood pressure of $140 / 80 \mathrm{~mm} \mathrm{Hg}$, pulse rate of $99 \mathrm{bpm}$, respiratory rate of 15 breaths per minute and temperature of $37.8^{\circ} \mathrm{C}$. On examination, there was an obvious swelling on the medial side of the right groin extending to the thigh with normal skin colour and temperature. By palpating the swelling, a tender hard lump could be felt below the inguinal ligament which did not have cough impulse and was partially reducible. The rest of the abdominal examination was unremarkable and there was no sign of bowel obstruction of peritonitis. Blood tests were requested that showed normal full blood count and electrolytes-white cell counts of $6 \times 10^{9} / \mathrm{L}$ with normal neutrophil count.

\section{DIFFERENTIAL DIAGNOSIS}

The clinical picture of a tender right groin mass is commonly associated with an incarcerated or strangulated femoral or inguinal hernia, and the patient may be feverish and laboratory values can be atypical. De Garengeot hernia should be suspected in an elderly woman presenting with signs and symptoms of a strangulated hernia. The differential diagnosis should include, in addition to inguinal hernia, adnexitis, a varix node, ectasia of the Vena saphena magna, lipomas or other soft tissue tumours, lymphomas and hypostatic abscesses in retroperitoneal processes. ${ }^{6}$ In our case, the patient had already been diagnosed with De Garengeot hernia by her GP after having CT scan.

\section{TREATMENT}

The patient was kept nil by mouth and after establishing intravenous access, intravenous fluid started. She received intravenous antibiotics (metronidazole $500 \mathrm{mg}$, gentamicin $280 \mathrm{mg}$, amoxicillin $1 \mathrm{~g}$ ) along with analgesia and transferred to the theatre for emergent appendectomy and reduction of the femoral hernia. Under general anaesthesia, a modified McEvedy method was used and a transverse 

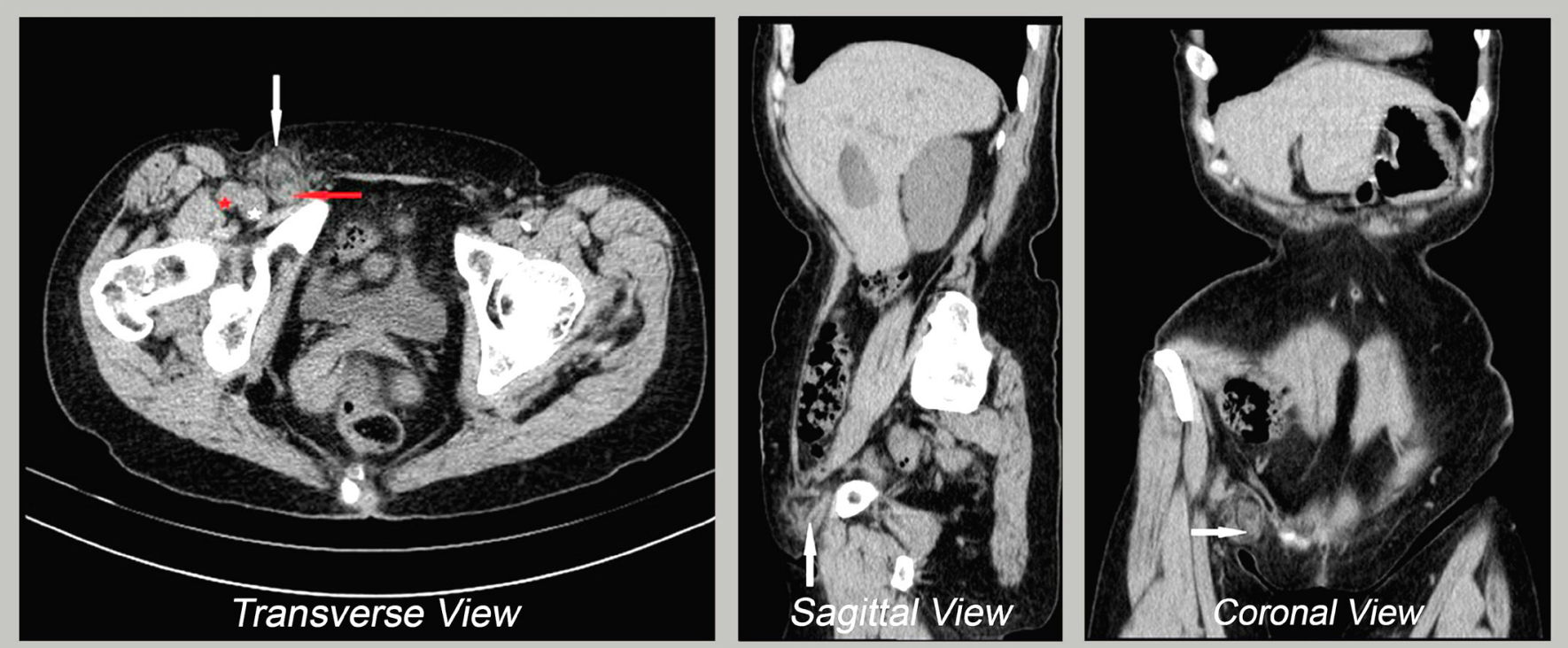

Figure 1 Abdominopelvic CT scan. Transverse view: inflamed appendix (red arrow) inside the femoral ring (white arrow), medial to the femoral vein (white asterisk) and femoral artery (red asterisk). Sagittal view: tubular structure inside the hernia sac. Coronal view: inflamed appendix with adjacent fat stranding.

incision $2 \mathrm{~cm}$ above the pubic tubercle was made; and after dissecting the rectus sheath, safe entry into the peritoneum was achieved. The lacunar ligament was carefully incised to allow reduction of the appendix. An inflamed appendix without signs of perforation was found and appendectomy was performed (figure 2). Afterwards, the femoral hernia repair was achieved by placing 1-0 Prolene suture between inguinal ligament and cooper ligament to fix the defect. Decision was made not to use mesh to reduce the risk of infection and peritoneum closed with 2-0 Vicryl and abdominal wall muscles closed with non-absorbable sutures. After closing the skin, local anaesthetic infiltrated around the incision line and patient transferred back to the ward.

\section{OUTCOME AND FOLLOW-UP}

She made an uneventful recovery and discharged the day after the procedure. A few days later, histopathology result came back which showed serosal and subserosal inflammation and fibroblastic proliferation in the appendix along with femoral

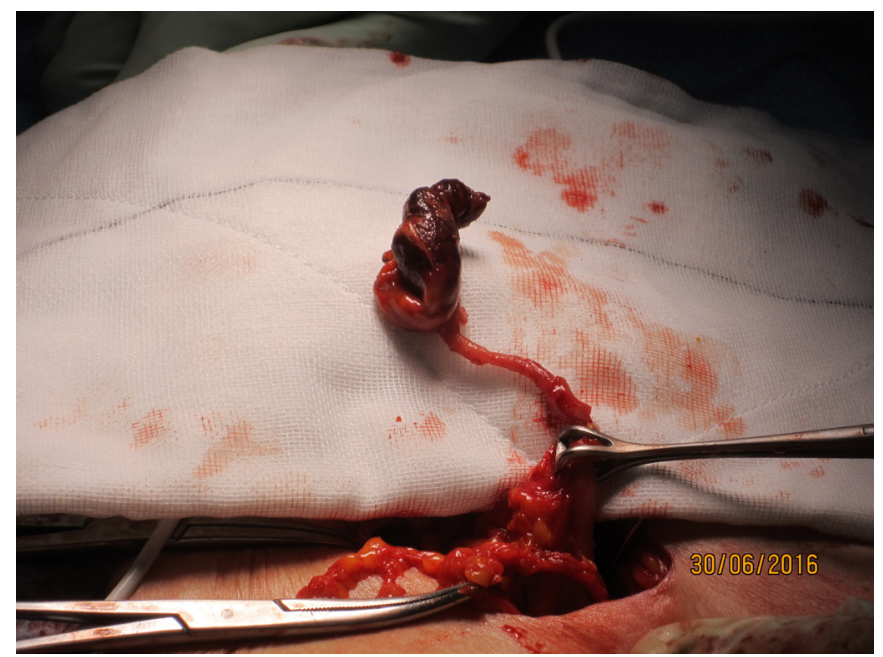

Figure 2 Intraoperative picture of the appendix with inflammatory changes. hernia sac contents. She was seen in the surgical outpatient clinic 2 weeks later and had a clean, well-healed surgical scar.

\section{DISCUSSION}

Rene Jacques Croissant de Garengeot was a French surgeon from Paris who first described De Garengeot hernia in $1731,{ }^{7}$ but it was Hevin who performed the first appendectomy on a patient with De Garengeot hernia in $1785 .^{8}$ Reflecting the higher incidence of femoral hernia in women, ${ }^{9}$ this type of hernia predominately affects postmenopausal women four times more, comparing to the men population. ${ }^{10}$ The median age for De Garengeot hernia is 55 years and risk factors for developing it are pregnancy-related changes, smoking, chronic cough, constipation and older age. ${ }^{10}$ There are two main theories to explain this condition. The first one emphasises on abnormal attachment of appendix to the caecum secondary to embryogenic rotations, and the second one claims that a large caecum can push the appendix into the femoral hernia sac. ${ }^{11}$ The sequence of the presence of appendicitis inside femoral sac is also controversial and unclear whether appendicitis happens first and then it migrates into the femoral hernia, or appendicitis occurs while the appendix had already engaged inside the femoral hernia. Followers of the latter scenario advocate that extraluminal compression of the appendix is more likely because of narrow neck of femoral hernia. ${ }^{4}$

De Garengeot hernia clinically presents with a groin lump (often erythematous) which is irreducible and tender to touch. Patient can have fever with signs and symptoms of bowel obstruction or shock. ${ }^{6}$ Sepsis and peritonitis are relatively uncommon because of the anatomical structure of the femoral canal which means that perforation is confined to this area and not spilled over to the peritoneal cavity. ${ }^{12}$

Most of the times, De Garengeot hernia is diagnosed intraoperatively and there are few cases that imaging already led to establishing diagnosis preoperatively. ${ }^{13}$ Abdominal X-ray does not help in detecting the hernia but is a useful tool if bowel obstruction is suspected. ${ }^{14}$ While ultrasound can reveal the bowel contents in hernia sac, ${ }^{15} \mathrm{CT}$ scan remains the gold standard of diagnosis. The findings of a low-lying caecum in combination with a blind-ended 
tubular structure inside the femoral hernia sac and stranding of nearby fat on CT scan has $98 \%$ sensitivity and specificity for diagnosing or excluding appendicitis within a hernia sac. ${ }^{16}$ MRI has comparable sensitivity and specificity to CT scan and can be requested for patients who are allergic to contrast. ${ }^{17}$

Treatment of De Garengeot hernia is an emergency. After initial resuscitation with intravenous fluid, antibiotics and analgesia, the patient is transferred to the operation room. Due to the rarity of this condition, there is no specific guideline as for surgical procedure. Lockwood-low incision, Lotheissen transinguinal incision, and McEvedy high incision are the main open approaches for repairing the femoral hernia. Decision on choosing the most suitable approach depends on patient's condition and also surgeon's opinion. In Lockwood-low incision (infrainguinal approach), which is the preferred method for elective repair of the hernia, femoral canal can be accessed by making an oblique incision $1 \mathrm{~cm}$ parallel and below the inguinal ligament. This approach has difficulties if there is bowel involvement, but is a simple method and abdominal wall is dissected less. ${ }^{18}$ The Lotheissen transinguinal approach involves making an incision superior and parallel to the inguinal ligament and extending from pubic tubercle to midinguinal point. In this type of incision, which is the favourable method when the diagnosis of inguinal and femoral hernia is in doubt, the inguinal canal is exposed as of the femoral hernia. Development of inguinal hernia in the future is the disadvantage of this procedure. ${ }^{18}$ The McEvedy high approach involves an oblique skin incision $3 \mathrm{~cm}$ above the pubic tubercle running laterally to cross the lateral border of the rectus muscle that is divided allowing preperitoneal dissection of the sac. This approach is preferred in the emergency setting when strangulation is suspected allowing better access to and visualisation of bowel for possible resection and can easily be converted to Pfannenstiel if full laparotomy is needed. ${ }^{18}$ The Laparoscopic approach for fixing De Garengeot hernia is still controversial. ${ }^{19} \mathrm{~A}$ laparoscopic approach with appendectomy followed by totally extraperitoneal procedure for femoral hernia repair was first reported by Beysens et al..$^{20}$ Some authors have recommended the laparoscopic approach for the diagnosis and repair of groin hernias with an atypical presentation or when the contents of the hernia cannot be determined using clinical or radiological exams, ${ }^{21}$ and the advantage of this approach is short hospital stay and fast recovery. ${ }^{22}$

The main complications of De Garengeot hernia repair, which are more common in elderly patients, are wound infection with the prevalence of $14 \%-29 \%$ and rarely necrotising fasciitis or death. ${ }^{23}$ It is found that delay in diagnosis, poor nutritional status,

Learning points

- De Garengeot hernia is a rare type of hernia which is the presence of appendix inside a femoral hernia sac.

- Patients usually present with signs and symptoms consistent with an incarcerated/strangulated femoral hernia and surgeons should be aware of it as one of the differential diagnosis of right lower quadrant abdominal pain.

- Preoperative diagnosis of De Garengeot hernia can be achieved with modern imaging such as ultrasound or CT scan.

- Surgery (appendectomy and herniorrhaphy) is the mainstay of treatment and should be done once diagnosed to prevent the complications. older age and multiple tissue planes involved in the repair of the hernia are contributing factors to increase the incidence of postoperative infections. ${ }^{14}$ In our case, patient had an uneventful recovery period and did not develop wound infection.

Contributors BB involved in collecting patient's images, obtaining the consent and drafting the manuscript. CLT was the operating surgeon and did the final edit and review.

Competing interests None declared.

Patient consent Obtained.

Provenance and peer review Not commissioned; externally peer reviewed.

Open Access This is an Open Access article distributed in accordance with the Creative Commons Attribution Non Commercial (CC BY-NC 4.0) license, which permits others to distribute, remix, adapt, build upon this work non-commercially, and license their derivative works on different terms, provided the original work is properly cited and the use is non-commercial. See: http://creativecommons.org/ licenses/by-nc/4.0/

(c) BMJ Publishing Group Ltd (unless otherwise stated in the text of the article) 2017. All rights reserved. No commercial use is permitted unless otherwise expressly granted.

\section{REFERENCES}

1 Townsend C, Beauchamp R, Evers B, et al. Sabiston textbook of surgery. 20th edn: Elsevier, 2017. Chapter 44.

2 Konofaos P, Spartalis E, Smirnis A, et al. De Garengeot's hernia in a 60-year-old woman: a case report. J Med Case Rep 2011;5:258.

3 Rajan SS, Girn HR, Ainslie WG. Inflamed appendix in a femoral hernial sac: de Garengeot's hernia. Hernia 2009;13:551-3.

4 Akbari K, Wood C, Hammad A, et al. De Garengeot's hernia: our experience of three cases and literature review. BMJ Case Rep 2014;2014:bcr2014205031.

5 Ardeleanu V, Chicos S, Tutunaru D, et al. A rare case of acute abdomen: Garengeot hernia. Chirurgia 2013;108:896-9.

6 Piperos T, Kalles V, Al Ahwal Y, et al. Clinical significance of de Garengeot's hernia: a case of acute appendicitis and review of the literature. Int I Surg Case Rep 2012;3:116-7.

7 Talini C, Oliveira LO, Araújo ACF, et al. De Garengeot hernia: case report and review. Int J Surg Case Rep 2015:8:35-7.

8 Akopian G, Alexander M. De Garengeot hernia: appendicitis within a femoral hernia. Am Surg 2005;71:526-7.

9 Hair A, Paterson C, O'Dwyer PJ. Diagnosis of a femoral hernia in the elective setting. J R Coll Surg Edinb 2001;46:117-8.

10 Kalles V, Mekras A, Mekras D et al. De Garengeot's hernia: a comprehensive review. Hernia 2013;17:177-82.

11 Weir CD, Doan SJ, Lughlin V, et al. Strangulation of the appendix in a femoral hernia sac. Ulster Med J 1994;63:114-5.

12 Mizumoto R, Hendahewa R, Premaratne G. De Garengeot hernia-Use of a novel surgical approach and literature review. Int J Surg Case Rep 2016;19:127-30.

13 Erdas E, Sias L, Licheri S, et al. De Garengeot hernia with acute appendicitis. G Chir 2013;34:86-9.

14 Nguyen ET, Komenaka IK. Strangulated femoral hernia containing a perforated appendix. Can J Surg 2004;47:68-9.

15 Filatov J, Ilibitzki A, Davidovitch S, et al. Appendicitis within a femoral hernia: sonographic appearance. J Ultrasound Med 2006;25:1233-5.

16 Pan CW, Tsao MJ, Su MS. A case of De Garengeot hernia requiring early surgery. BMJ Case Rep 2015;2015:bcr2015211102.

17 Halpenny D, Barrett R, O'Callaghan K, et al. The MRI findings of a de Garengeot hernia. Br J Radio/ 2012;85:e59-61.

18 Sorelli PG, El-Masry NS, Garrett WV. Open femoral hernia repair: one skin incision for all. World J Emerg Surg 2009;4:44.

19 Al-Subaie S, Mustafa H, Al-Sharqawi N, et al. A case of de Garengeot hernia: the feasibility of laparoscopic transabdominal preperitoneal hernia repair. Int J Surg Case Rep 2015;16:73-6.

20 Beysens M, Haeck L, Vindevoghel K. Laparoscopic appendectomy combined with TEP for de Garengeot hernia: case report. Acta Chir Belg 2013;113:468-70.

21 Al-Subaie S, Mustafa H, Al-Sharqawi N, et al. A case of de Garengeot hernia: the feasibility of laparoscopic transabdominal preperitoneal hernia repair. Int J Surg Case Rep 2015;16:73-6

22 Comman A, Gaetzschmann P, Hanner T, et al. DeGarengeot hernia: transabdominal preperitoneal hernia repair and appendectomy. JSLS 2007;11:496-501.

23 Sharma H, Jha PK, Shekhawat NS, et al. De Garengeot hernia: an analysis of our experience. Hernia 2007;11:235-8. 
Copyright 2017 BMJ Publishing Group. All rights reserved. For permission to reuse any of this content visit http://group.bmj.com/group/rights-licensing/permissions.

BMJ Case Report Fellows may re-use this article for personal use and teaching without any further permission.

Become a Fellow of BMJ Case Reports today and you can:

- Submit as many cases as you like

- Enjoy fast sympathetic peer review and rapid publication of accepted articles

- Access all the published articles

- Re-use any of the published material for personal use and teaching without further permission

For information on Institutional Fellowships contact consortiasales@bmjgroup.com

Visit casereports.bmj.com for more articles like this and to become a Fellow 